\title{
Extensional midline framework built with porous polyethylene implant (Medpor) in rhinoplasty
}

\author{
Chen Zhang, MD, PhD, Xiao-Li Jiang, MD, Chun-Ying Ge, MD, Li-Nan Song, MD \\ Institute of Plastic Surgery, Dalian University, Dalian, P.R. China
}

\begin{abstract}
Background: In East Asians, the main steps of rhinoplasty include the regulation of the nasal tip projection along with dorsal augmentation. A complete septal extension graft and columella strut graft are effective tools for the correction of unprojected tips and short noses. However, autologous cartilages cannot provide enough cartilage for the graft. Moreover, use of rib cartilages will leave an additional scar on the patient's chest, and these are not considered a common source of cartilage. Therefore, the authors used porous high-density polyethylene (Medpor) sheets to rebuild extensional midline framework in rhinoplasty.

Objective: To study the possibility and the method of porous high-density polyethylene (Medpor) sheets used as extensional midline framework in rhinoplasty.

Methods: From May 2012 to May 2016, 78 patients underwent primary rhinoplasty with a midline framework built with Medpor. The patients' ages ranged from 22 to 48 years (mean $\pm S D, 26 \pm 5.2$ years). Seventy-five patients were women, and three were men. The patients selected Medpor because of a lack of adequate autogenous septal cartilage and refusal to use rib cartilage.

Results: The patients were followed up for 6 months to 4 years, with a mean follow-up period of 18 months, in 3-month intervals, and examined for extrusion, infection, and aesthetic outcomes. In the 78 patients, 156 pieces of Medpor graft were used with 78 extensional septal grafts and 78 columella strut grafts. Nasal tip projection and columella-labial angle were changed significantly after the surgery in 67 of the 78 patients. Complications occurred in 11 patients, of whom one had two minor complications of deviated tip and columella.

Conclusion: The porous high-density polyethylene sheets are easier to handle and effective as materials to build an extensional midline framework in rhinoplasty. However, the size of the porous high-density polyethylene sheets should be tailored carefully based on the preoperative assessment; otherwise, severe complications will occur.
\end{abstract}

Keywords: porous polyethylene; implant; rhinoplasty; extensive spreader graft; columellar strut

\section{Introduction}

In East Asia, people have different facial profiles from Westerners owing to their different ethnicity and anatomy. Their nose is small with a low nasal radix, unprojected bubble tip, and wide base. Most East Asians prefer a higher radix and a more projected tip. In the past, the most popular rhinoplasty was the insertion of a silicone implant into a nasal dorsal pocket. However, if the caudal part of the silicone implant goes beyond the septal angle, the postoperative nasal tip projection will be in disharmony with the height of the nasal dorsum because the caudal part of the implant is short of enough support from the septum. Thus, the L-shape implant was used to replace the I shape implant to solve the problem. However, this led to anoth-

Received January 16 2017, Revised May 22 2017, Accepted May 242017

Corresponding author: Chen Zhang, Institute of Plastic Surgery, Dalian University, 3-2-1 Bldg 24, East Jinhai Garden, Xigang District, Dalian City, Liaoning 116021, P.R. China

Tel: 86-13998612400, Fax: 86-411-84369828, E-mail: neilzhang@sina.com

This is an Open Access article distributed under the terms of the Creative Commons Attribution Non-Commercial License (http://creativecommons.org/licenses/by-nc/4.0), which permits unrestricted non-commercial use, distribution, and reproduction in any medium, provided the original work is properly cited.

Copyright (C) 2017. Korean Society of Korean Cosmetic Surgery and medicine (KSKCS \& KCCS). 
er problem, an overtensioned tip. This means that the implant poked the soft tissues of the nasal tip, feeling and appearing as if it was going to protrude at any time. In the late 1990s, some basic concepts of rhinoplasty in the West were introduced into East Asia by Korean physicians. They were dorsal augmentation with implant and tip plasty with autonomous cartilages harvested from the septum, choncha, and rib [1-5].

Autogenous septal cartilage is generally accepted as the gold standard nasal grafting material; however, the amount of septal cartilage remaining is usually insufficient because of prior trauma, surgery, or infection. Other autogenous materials such as costal and conchal cartilages offer a bountiful supply of cartilage and can provide structural support but are associated with donor site morbidity. In a recent clinical research, Medpor was shown to be a useful dorsal and spreader graft in reconstruction of severe nose deformities, with the lowest complication rate and without infectious complications and extrusion [6-10]. Thus, building a midline framework with Medpor to extend the septum will provide a strong support to the caudal part of the implant and autogenous cartilage grafts on the tip.

\section{Materials and methods}

This was a retrospective study approved by the institutional review board of Xinhua Hospital, Dalian University. Medpor was first used in our division in June 2012, after a thorough literature review of the possible use of Medpor in rhinoplasty. The clinical data reviewed included rhinoplasty cases with Medpor from June 2012 to June 2016. Seventy-eight patients underwent primary rhinoplasty with a midline framework built with Medpor. Their ages ranged from 22 to 48 years (mean \pm SD, 26 \pm 5.2 years). Seventy-five patients were women, and three were men. The patients selected Medpor because of a lack of adequate autogenous septal cartilage and refusal to use rib cartilage. All advantages, disadvantages, and possible complications of Medpor were explained to the patients.

\section{Operative techniques}

In this series, operative techniques were based on the authors' previous research [11]. An open approach and an inverted $\mathrm{V}$ transcolumella incision were used in all the patients. After total degloving of the skin soft tissue envelope from the lower lateral cartilages, the interdomal ligament was separated and cut in half. Thereby, the septal angle was unveiled, and then the dorsal side of septal cartilage was dissected from its mucopericondrium at approximately $5 \times 30 \mathrm{~mm}$ and to form the cavities for Medpor implants. An ultrathin Medpor sheet $(0.85 \mathrm{~mm})$ was cut into $3 \times 30-\mathrm{mm}$ pieces, and the Medpor stripes were inserted into the pockets between the septal cartilage and its mucopericondrium. To fix the Medpor implant and septum together, 6-0 PDS was used with interrupted horizontal sutures. The part of Medpor beyond the septal angle was then covered by the interdomal ligament, and then they were sutured together. After the sutures, the dome of the nose could be moved nearly $5 \mathrm{~mm}$ caudally, which means that the nose was lengthened by $5 \mathrm{~mm}$. If some part of Medpor remains over the cephalic margin of the medial crura, the extra part was cut off.

Then, a $3 \times 30$-mm pocket was separated between the medial crura and columella strut of Medpor, and inserted between the medial crura and secured with through-and-through 4-0 Monocryl sutures at the base, subdome, and region of the medial crura. The extra part of Medpor over the base of the dome was also cut off.

At this time, the new midline framework was completed and ready for supporting its overlying implant and grafts (Fig. 1). The rest of the steps of the operation were the insertion of a silicone or Gore Tex implant, and tip grafts for tip plasty as in the standard rhinoplasty. For grafts on the tip, both onlay and shield grafts are necessary to refine the shape of the tip and to cover Medpor.

\section{Results}

In this study, the patients were followed up for 6 months to 4 years, with a mean follow-up period of 18 months, in 3-month intervals, and examined for extrusion, infection, and aesthetic outcomes. In the 78 patients, 156 pieces of Medpor graft were used, with 78 extensional septal grafts and 78 columella strut grafts. Nasal tip projection and columella-labial angle were changed significantly after the operation in 67 of the 78 patients (Figs. 2 4).

Complications occurred in 11 patients, of whom one had two minor complications of deviated tip and columella. Severe complications included columellar incision dehiscence and fistula formation in 4 cases (Fig. 5) and implant pressure on the columella in 1 case (Fig. 6). For incision dehiscence, the columellar strut was shortened, and the incision healed uneventfully in one case. However, in the other 3 cases, the incision failed to close and formed fistula after 1 month of irrigation with gentamycin saline, which was resolved by removal of all implants. For the case with implant pressure on the columella, part of the extensional septal graft was cut off and the columella 

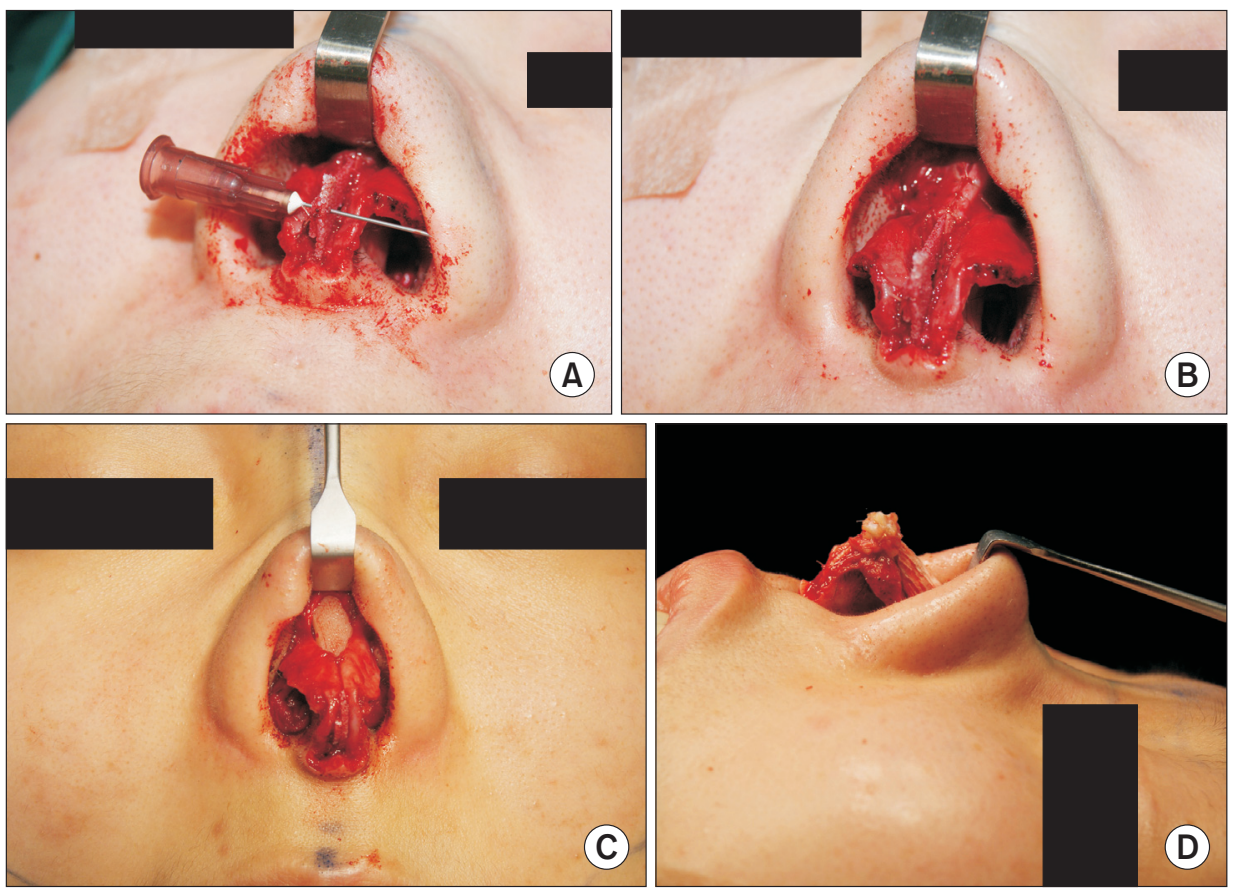

Fig. 1. Extensional midline framework built with porous polyethylene implant (Medpor) for cartilage grafts. (A) Medpor ESG is sutured in place, and a Medpor columellar strut is placed between the medial crus. (B) Extensional midline framework built with porous polyethylene implant (Medpor) is ready for cartilage grafts. (C) Front view showing the relationship among the Medpor framework, the implant (silicone), and the lower lateral cartilage. (D) Lateral view showing that after the Medpor framework was fabricated, the relationship among the cartilage graft, the implant (ePTFE), and Medpor framework.
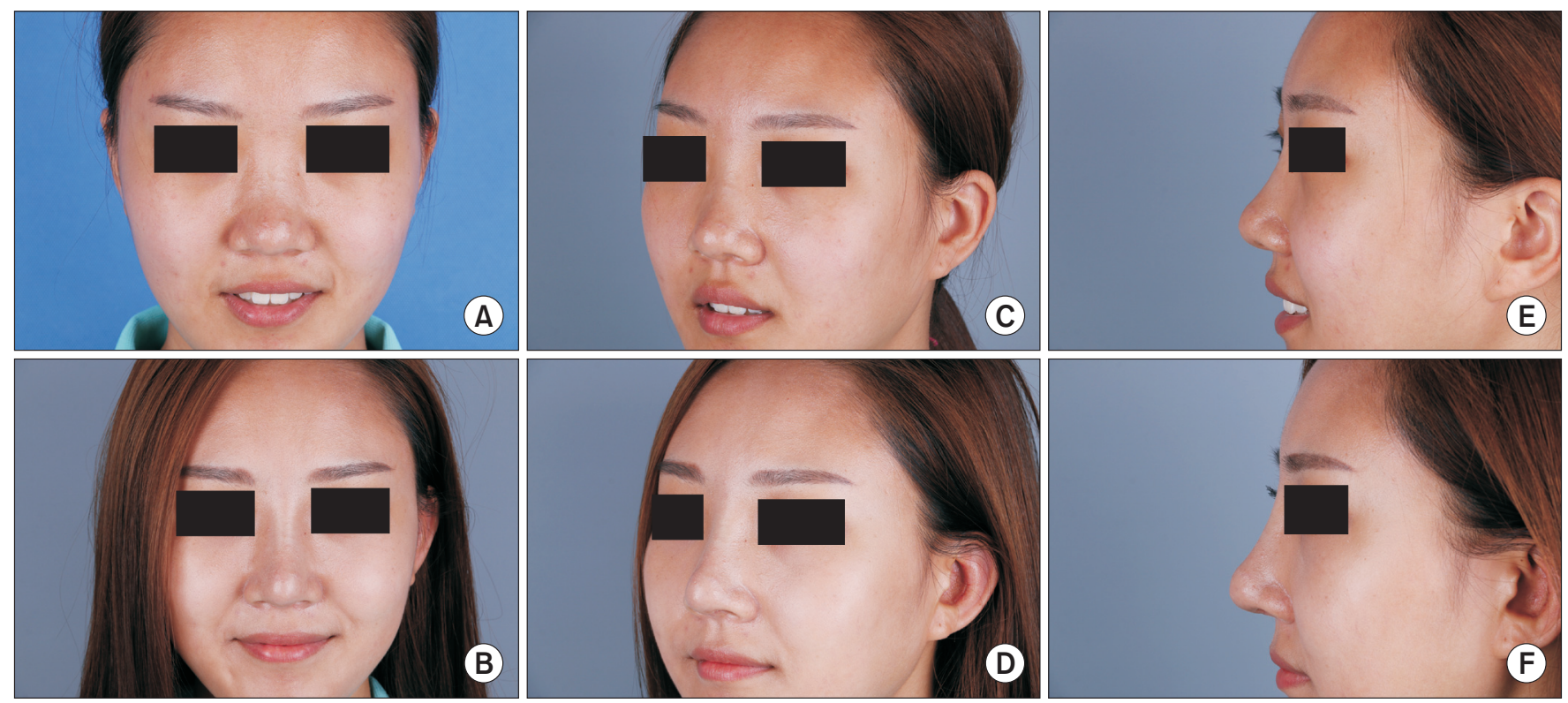

Fig. 2. A 21-year-old girl complained of low nasal bridge and nasal tip. She had a hyaluronic acid injected on her bridge and sought for rhinoplasty. Medpor was used to extend her septum, and then onlay and shield grafts were used to regulate tip projection. Expanded polytetrafluoroethylene was used for nasal dorsal augmentation. (A, C, E) Preoperative photos. (B, D, F) Six-month postoperative photographs.

was restored. The minor complications were clicking sound when the patients pressed on their nasal tips ( 2 cases), and deviated tip ( 3 cases) and columella ( 2 cases). For the former two patients, they did not want a revision after receiving an explanation of the reasons for the sound. For the latter patients, because the complications were found in the early days after the operation, they were treated with conservative methods, including stripe paper tape and soft nostril tube support. 

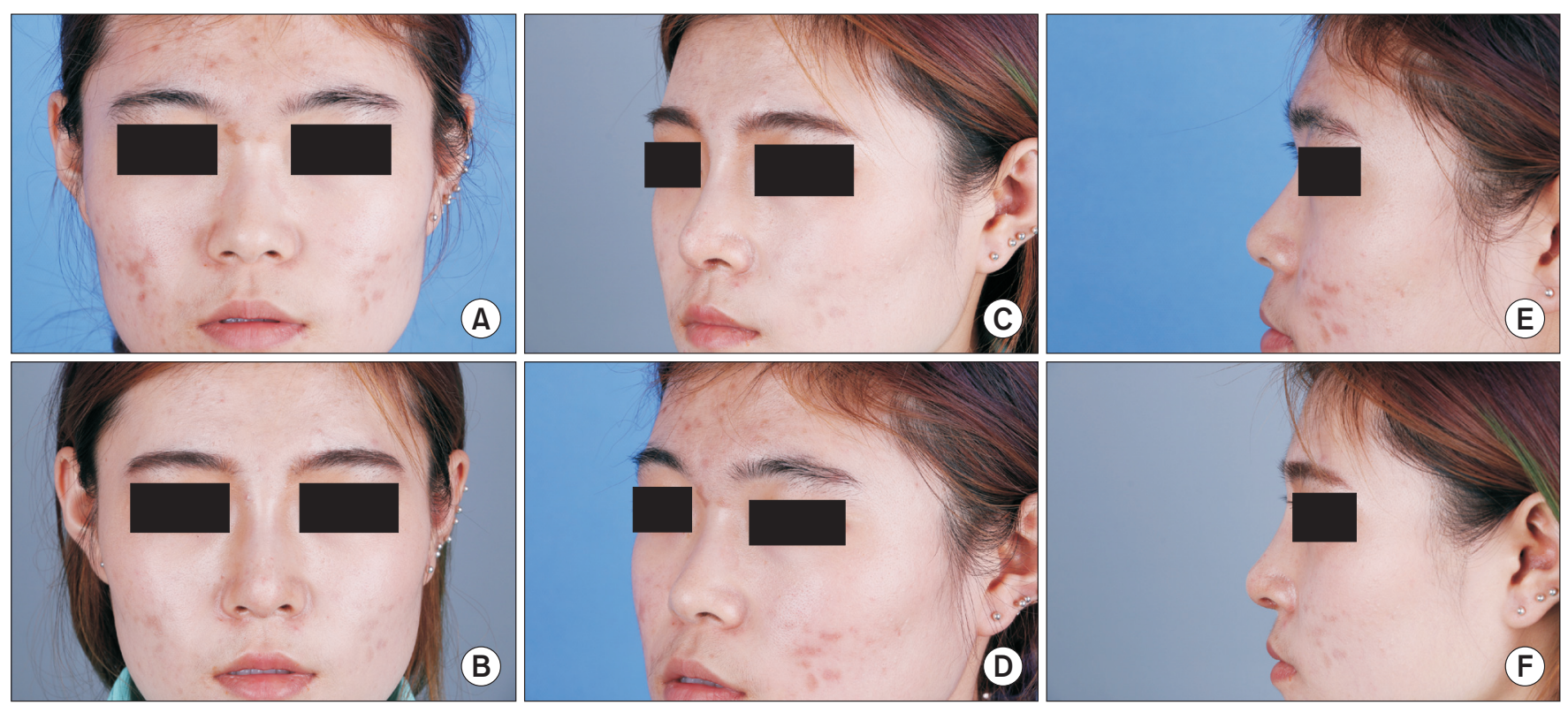

Fig. 3. This 24-year-old patient had a rhinoplasty with Medpor implant sheets and ear cartilage graft. Silicone implant was used for the nasal dorsal augmentation. (A, C, E) Preoperative photographs. (B, D, F) Six-month postoperative photographs.

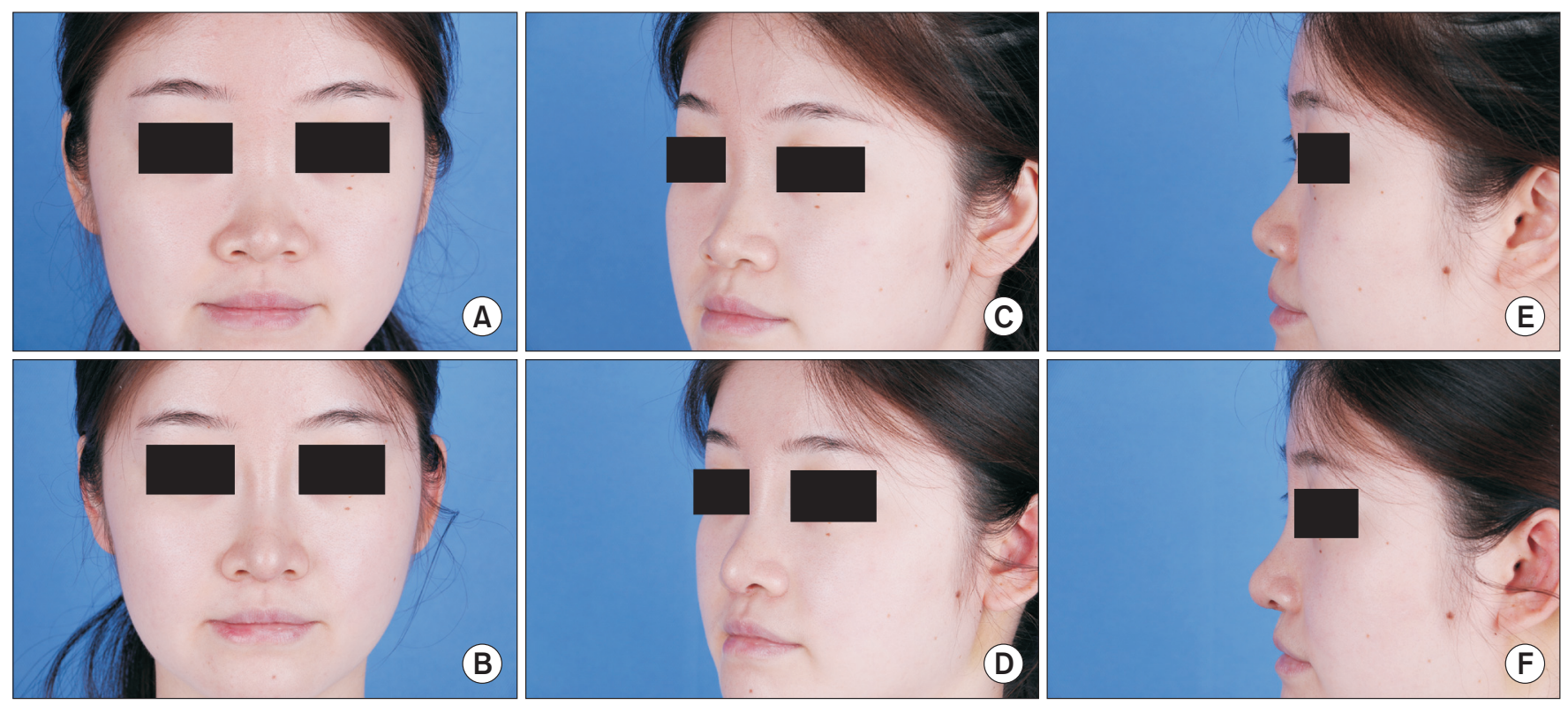

Fig. 4. Medpor implant sheets, and an ear cartilage graft and ePTFE were used for rhinoplasty. (A, C, E) Preoperative photographs. (B, D, F) Thirteen-month postoperative photographs.

\section{Discussion}

Rhinoplasty in East Asia means augmentation rhinoplasty by making the nose longer, and the nasal radix and tip higher. Making the radix higher is easy with artificial implant because of the thicker soft tissue envelop in East Asians than in Westerners. However, changing the tip into a more projecting position is not easy. The tip position, which is a tip-defining point, is the position of the dome maintained by the lower lateral cartilage in a sophisticated way such that the medial and lateral crura act as a tripod. Thus, the tip position change can be realized by dome position change and application of grafts to the dome. No matter the method used, the basic condition is to build a stable base to support the dome and the grafts on it. 


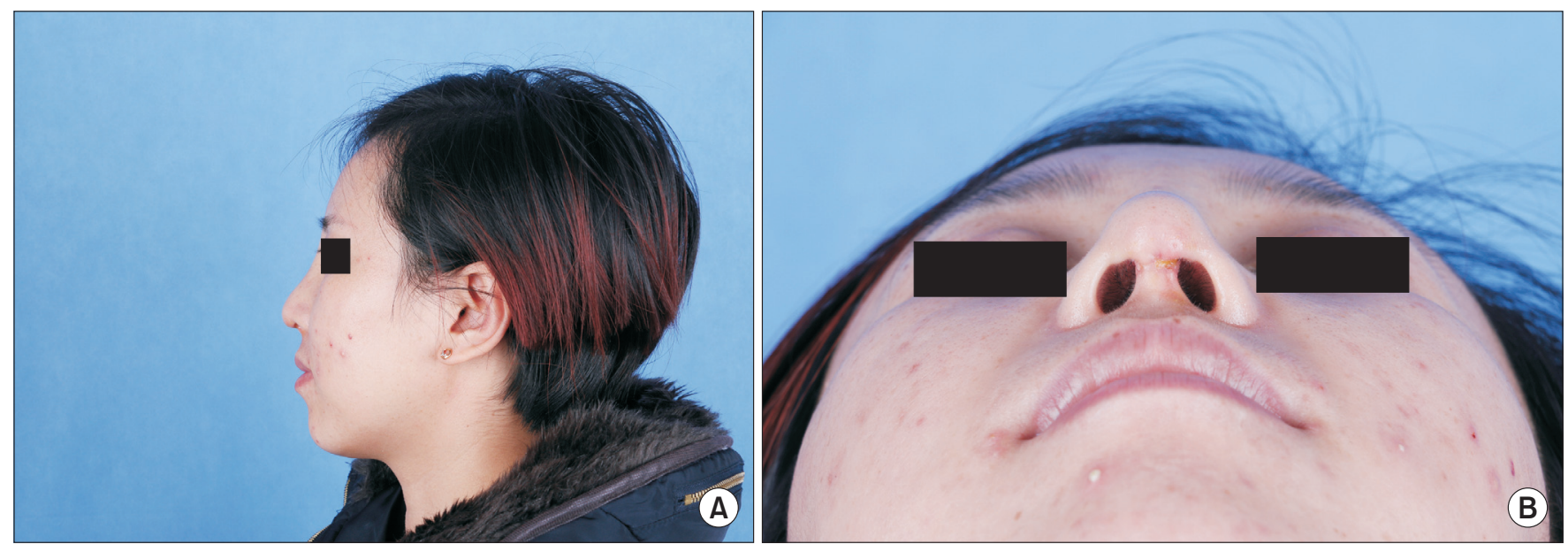

Fig. 5. For this patient, the incision failed to close and formed a fistula after 1-month irrigation with gentamycin saline, which was resolved by removal of all implants.
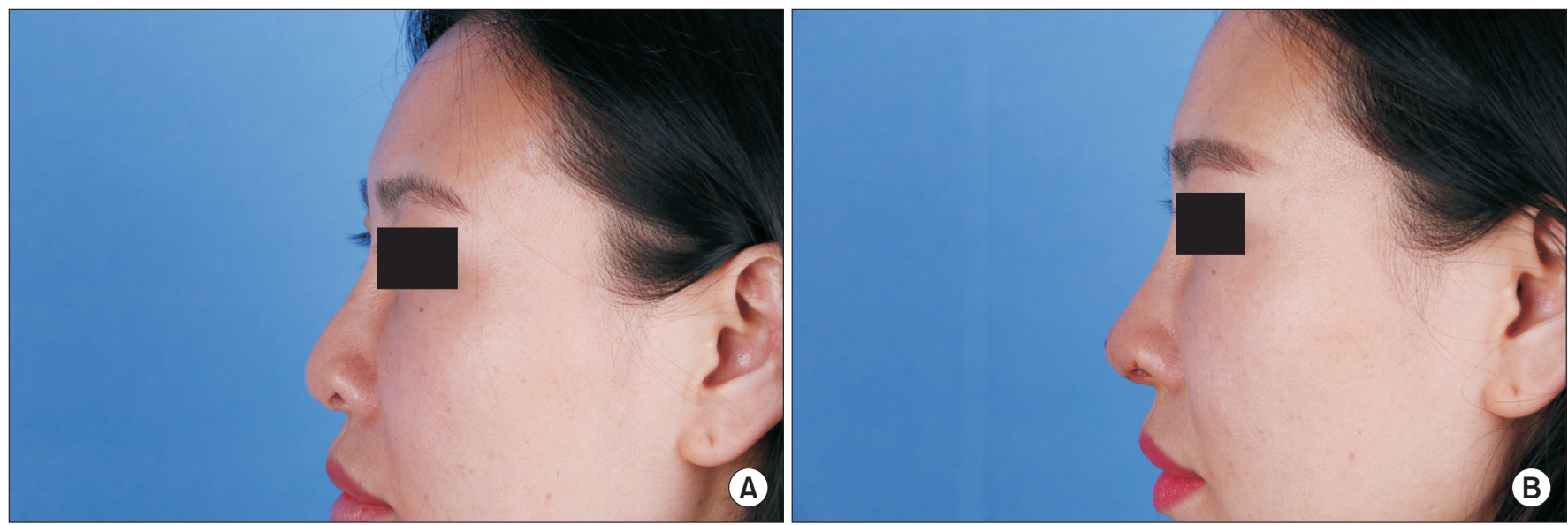

Fig. 6. A case with implant pressure on the columella. Part of the extensional septal graft was cut off, and the columella was restored.

To provide the nasal tip with a strong support, the septal angle should be extended cephalically. The useful tool was the septum extensional graft in the horizontal direction and columella strut in the anteroposterior direction. In the earlier cases, the two parts were sutured together. However, the fixed two parts make the tip rigid in texture and moved less. Thus, the Medpor grafts were cut in short and not fixed together to give the dome more cavities to move freely.

Medpor can be used as a graft of different sizes with high biocompatibility that allows for specific use in the reconstruction of deviated noses, saddle deformities, and revision surgery rhinoplasty due to lack of cartilage [12-14]. However, nowadays use of alloplastic implants, especially Medpor, has decreased because of reports of complications such as extrusion and fistula $[15,16]$. Although most surgeons prefer autologous grafts, graft selection must be individualized for each patient to take into account history of prior surgery, associated structural abnormalities, and characteristics of the overlying skin and soft tissue.

Medpor implants have pores with sizes ranging from 100 to $250 \mathrm{~mm}$ (mean, $150 \mathrm{~mm}$ ). In a follow-up study, Neel [17] found that porous polyethylene implants with $145-\mathrm{mm}$ pores were quickly infiltrated by fibrovascular tissue but also noted a high extrusion rate when the implants were placed in subcutaneous pockets in the rabbit ear. Wellisz and Dougherty [18] reported 17 nasal alar implants in burn patients; 2 implants became exposed, and 1 was removed. Recently, Romo III et al. described 187 cases of functional and aesthetic nasal reconstructions using Medpor implants. Indications for Medpor implant nasal reconstruction included prior rhinoplasty with nasal valve collapse and aesthetic rhinoplasty of the platyrrhine nose. They 
have used Medpor implants for nasal dorsal and tip augmentation, columellar struts, alar battens, and premaxillary plumper implants [19]. Mohammadi et al.[7] showed that minimum complications were low when Medpor implants were used as dorsal and, especially, spreader grafts, but most of the infectious complications and extrusion were observed after implanting Medpor as a strut and rim graft. They recommend the safe use of Medpor implants only as spreader graft.

In our series, complications occurred in 11 patients, of whom one had two minor complications of deviated tip and columella. Severe complications included columellar incision dehiscence (4 cases) and implant pressure on the columella (1 case). All these complications occurred in the early cases. We believe that they were results from over-lengthened Medpor implants. After the procedures such as covering the caudal part of Medpor with interdomal ligament were improved and the Medpor size changed, less similar complications occurred in the later cases. The minor complications were the clicking sound when the patients pressed on their nasal tips (2 cases), and deviated tip (3 cases) and columella (2 cases). For the former two patients, they did not want a revision after receiving an explanation of the reasons for the sound. For the latter, because the complications were found in the early days after operation, they were treated with conservative methods, including stripe paper tape and soft nostril tube support.

\section{Conclusion}

In summary, the porous high-density polyethylene sheets are easier to handle and effective as material with which to build an extensional midline framework in rhinoplasty. However, the sizes of porous high-density polyethylene sheets should be tailored carefully on the basis of the preoperative assessment. Otherwise, some severe complications such as extrusion and incision dehiscence will occur.

\section{Conflicts of interest}

The authors have nothing to disclose.

\section{References}

1. Lee Y, Kim J, Lee E. Lengthening of the postoperative short nose: combined use of a gull-wing concha composite graft and a rib costochondral dorsal onlay graft. Plast Reconstr Surg 2000;105:2190-9.
2. Park HS. Lambda-shaped implant for augmentation of anterior nasal spine in Asian rhinoplasty as an ancillary procedure. Aesthetic Plast Surg 2001;25:8-14.

3. Jung DH, Kim HJ, Koh KS, Oh CS, Kim KS, Yoon JH, et al. Arterial supply of the nasal tip in Asians. Laryngoscope 2000;110:308-11.

4. Lee HM, Kang HJ, Choi JH, Chae SW, Lee SH, Hwang SJ. Rationale for osteotome selection in rhinoplasty. J Laryngol Otol 2002;116:1005-8.

5. Han SK, Lee DG, Kim JB, Kim WK. An anatomic study of nasal tip supporting structures. Ann Plast Surg 2004;52:134-9.

6. Scopelliti D, Amodeo G. Nasal dorsum reconstruction with alloplastic material. J Craniofac Surg 2016;27:e676-8.

7. Mohammadi S, Mohseni M, Eslami M, Arabzadeh H, Eslami M. Use of porous high-density polyethylene grafts in open rhinoplasty: no infectious complication seen in spreader and dorsal grafts. Head Face Med 2014;10:52.

8. Zhou J, Huang X, Zheng D, Li H, Herrler T, Li Q. Oriental nose elongation using an L-shaped polyethylene sheet implant for combined septal spreading and extension. Aesthetic Plast Surg 2014;38:295-302.

9. Jang D, Yu L, Wang Y, Cao D, Yu Z, Mu X. Nasal measurements in Asians and high-density porous polyethylene implants in rhinoplasty. Arch Facial Plast Surg 2012;14:181-7.

10. Han K, Jeong JW, Kim JH, Son D, Kim S, Park SW, et al. Complete septal extension grafts using porous high-density polyethylene sheets for the westernization of the Asian nose. Plast Reconstr Surg 2012;130:106e-15e.

11. Wang M, Jiang XL, Zhang C, Ge CY, Wang JQ, Yang W, et al. Application of Medpor implant on tip rhinoplasty. Chin J Aesthet Plast Surg 2015;26:324-26.

12. Emsen IM. E-M shaped septal encircling with Medpor reconstruction on crooked noses: personal technique and postoperative results. J Craniofac Surg 2008;19:216-26.

13. Romo T 3rd, Kwak ES. Nasal grafts and implants in revision rhinoplasty. Facial Plast Surg Clin North Am 2006;14:373-87, vii.

14. Romo T 3rd, Sclafani AP, Sabini P. Reconstruction of the major saddle nose deformity using composite allo-implants. Facial Plast Surg 1998;14:151-7.

15. Alonso N, de Pochat VD, de Barros AR, Tavares LS. Long-term complication after rhinoplasty using porous polyethylene implant: cutaneous fistula of the forehead. J Craniofac Surg 2013;24:2176-8.

16. Seyhan T, Borman H, Deniz M, Kocer E. Intranasal porous polyethylene implant extrusion 7 years after insertion in a pa- 
tient with Hashimoto disease. J Craniofac Surg 2009;20:73-4.

17. Neel HB. Implants of Gore-Tex comparisons with Tefloncoated polytetrafluoroethylene carbon and porous polyethylene implants. Arch Otolaryngol 1983;109:427-33.

18. Wellisz T, Dougherty W. The role of alloplastic skeletal modi- fication in the reconstruction of facial burns. Ann Plast Surg 1993;30:531-6.

19. Romo T 3rd, Kwak ES, Sclafani AP. Revision rhinoplasty using porous high-density polyethylene implants to reestablish ethnic identity. Aesthetic Plast Surg 2006;30:679-84. 